\title{
Technical evaluation of a third generation optical pose tracker for motion analysis and image-guided surgery
}

\author{
Juan A. Sánchez-Margallo ${ }^{1}$, Francisco M. Sánchez-Margallo ${ }^{1}$, José B. Pagador ${ }^{1}$, Ignacio \\ Oropesa $^{2}$, Marcos Lucas ${ }^{1}$, Enrique J. Gómez ${ }^{2}$, José Moreno ${ }^{3}$ \\ ${ }^{1}$ Jesús Usón Minimally Invasive Surgery Centre, Cáceres, Spain \\ \{jasanchez, msanchez, jbpagador, mlucas\}@ ccmijesususon.com \\ ${ }^{2}$ Bioengineering and Telemedicine Centre (GBT), ETSI Telecomunicación, Universidad \\ Politécnica de Madrid, Madrid, Spain; Networking Research Center on Bioengineering, \\ Biomaterials and Nanomedicine (CIBER-BBN), Zaragoza, Spain \\ \{ioropesa, egomez\}@gbt.tfo.upm.es \\ ${ }^{3}$ Laboratory of Robotics and Artificial Vision, University of Extremadura, Cáceres, Spain \\ josemore@unex.es
}

\begin{abstract}
Laparoscopic instrument tracking systems are an essential component in image-guided interventions and offer new possibilities to improve and automate objective assessment methods of surgical skills. In this study we present our system design to apply a third generation optical pose tracker (MicronTracker®) to laparoscopic practice. A technical evaluation of this design is performed in order to analyze its accuracy in computing the laparoscopic instrument tip position. Results show a stable fluctuation error over the entire analyzed workspace. The relative position errors are $1.776 \pm 1.675 \mathrm{~mm}$, $1.817 \pm 1.762 \mathrm{~mm}, 1.854 \pm 1.740 \mathrm{~mm}, 2.455 \pm 2.164 \mathrm{~mm}, 2.545 \pm 2.496 \mathrm{~mm}$, $2.764 \pm 2.342 \mathrm{~mm}, 2.512 \pm 2.493 \mathrm{~mm}$ for distances of 50, 100, 150, 200, 250, 300, and $350 \mathrm{~mm}$, respectively. The accumulated distance error increases with the measured distance. The instrument inclination covered by the system is high, from 90 to 7.5 degrees. The system reports a low positional accuracy for the instrument tip.
\end{abstract}

Keywords. Laparoscopic tool tracking, Optical pose tracker, Motion analysis, Image-guided surgery

\section{INTRODUCTION}

The benefits of minimally invasive surgery (MIS) for patients, such as less pain, less scarring, and less postoperative periods, have made these surgical techniques the focus of attention in recent years. However, the evolution from open surgery to MIS requires learning and training new surgical manoeuvres and the way to use a new set of surgical tools.

Nowadays, there are several platforms, devices, and live models that make acquisition of these new cognitive and psychomotor skills easier [1-3]. Virtual simulators provide different surgical scenarios and software to assess surgical practice. However, 
the realism of the haptic sense is still poor and they are not always affordable for all surgical training centres or hospitals. In some cases, the use of animal models can be replaced by inanimate systems, like physical simulators, which are a very economically reasonable way to train fundamental surgical tasks and procedures with a completely real haptic sensation. Nevertheless, their assessment methods are often basic, subjective, or require the interaction of an experienced surgeon.

Surgical training has seen a positive development from traditional Halsted-based surgical training method [4] to more reliable objective procedures. Several objective assessment methods based on structured checklist and end-product analysis have been proposed [5]. However, some drawbacks, like the high resources required, the possible ambiguity of their scores, and the need of the presence of an experienced supervisor, drive the necessity to develop more automated evaluation systems.

Psychomotor analysis has been used successfully in several studies to address the assessment of surgeons' skills [6]. Different technologies have been applied to implement objective assessment systems that make use of this criterion. Electromagnetic devices have been attached to the laparoscopic instruments to measure their movements. Some of their features, like size and precision, have evolved in recent years, but they have still significant interference problems. Optical systems make use of markers to be detected by a camera system providing a high accuracy, but they need a clear line of sight. Another approach uses the laparoscope as information source. Computer vision-based methods are based on probabilistic techniques, shape of the instrument or artificial markers placed on the instruments. Most of these systems however have difficulties with the real-time execution and their positional accuracy.

Besides their use as tools for motion analysis, tracking devices are an essential component of image-guided surgery systems. Instrument tracking is useful to the surgeon when the surgical instrument is outside the field of view, when it is difficult to identify in the image due to the artefact that it produces, or when the instrument cannot be readily detected by the imaging modality [7].

We propose to design a system in order to apply a third generation optical pose tracker (MicronTracker® 3 Hx60; Claron Technology Inc., Toronto, CAN) for objective assessment of surgical skills based on motion analysis and for image-guided interventions. This system has been adapted to be used with real laparoscopic instruments.

Optical pose trackers of the first-generation use light-emitting targets, such as LEDs. Second-generation sensors emit light from a ring surrounding each camera lens and typically use balls or discs as targets coated with retro-reflective material. Both first and second-generation trackers cannot guarantee the visibility of targets under visible light conditions; hence infrared (IR) light is used. The intensity of the IR light falls with the square of the distance and to prevent sensor saturation both kinds of trackers do not allow targets to be placed close to the camera. This leads to larger cameras being needed to obtain the required accuracy. Nevertheless, it increases the possibility of line-of-sight interruptions and makes the positioning of the camera more difficult, especially when space is crucial.

In this study we present our system design to apply the use of a third generation pose tracker to laparoscopic practise. We tested the positional accuracy of the system 
in computing the laparoscopic instrument tip. This is the first step to use this system as a reliable tool to track the laparoscopic instrument during the surgical practice, providing the required information in order to perform psychomotor analysis of instruments motion and be used in image-guided surgical applications.

\section{$2 \quad$ Material and methods}

\subsection{System description}

A support with three different markers was designed for each laparoscopic instrument. The pattern of the markers was geometrically defined and arranged according to the rules dictated by the tracker's designers. We used three markers to have a frontal and two lateral views of the instrument, and therefore control the possible loss of any marker's position. To perform this study we have used a laparoscopic needle holder, but this procedure can be extended to other kind of instruments.

Two different supports, one for laparoscopic scissors, dissectors, and graspers; and another one for straight and curved needle holders were designed to hold the three markers on the instruments (Figure 1). They have been integrated to ensure the natural use and gripping of the instruments and they have been made of Polyvinyl chloride (PVC) in order to provide lightweight and tough pieces.

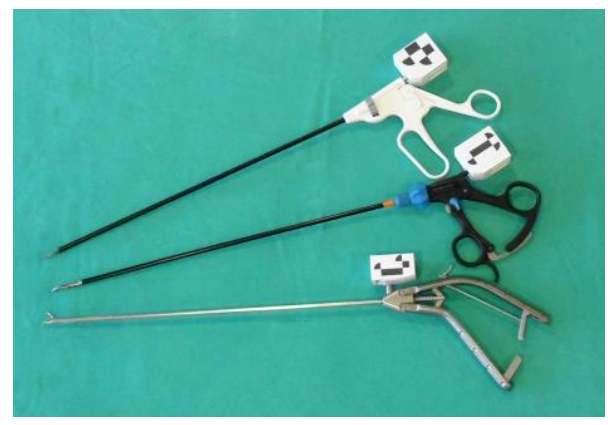

Fig. 1. Set of laparoscopic instruments with their marker supports.

The transformation matrices from the markers to the tool tip were computed using a calibration plate placed under the tip of the laparoscopic instrument. With this information it was possible to project each marker position on the instrument to the three-dimensional position of the tip.

The camera system has an electrical interface IEEE-1394a. A software application running on a Linux-based computer detects the visible targets in the image, its positions, and its projection at the tip of the instrument.

In accordance with the technical specifications of the manufacturer, the camera has a calibration error $<0.35 \mathrm{~mm}$ RMS, a jitter error with static target $<0.015 \mathrm{~mm}$ RMS and a jitter error with moving target $<0.14 \mathrm{~mm}$ RMS. These errors are computed for a 
single target at a distance of $60-170 \mathrm{~cm}$ for the calibration error and at $75 \mathrm{~cm}$ for both jitter errors.

\subsection{Technical validation}

The first step was to test the system in a physical laparoscopic simulator. Therefore, the tracker was placed in front of the simulator at $600 \mathrm{~mm}$ from it. The workspace volume for the laparoscopic simulator was $500 \times 450 \times 600 \mathrm{~mm}$ (length $\mathrm{x}$ width $\mathrm{x}$ height). This was the reference volume to perform the accuracy tests of the system. The high of the camera system was approximately the same as the high of the markers support on the instrument $(340 \mathrm{~mm})$. The workspace volume has been established within the field of measurement defined by the manufacturer as the volume in which single targets of $12 \mathrm{~mm}$ radius can be detected within its accuracy specifications.

Several tests were performed to verify the positional accuracy of the system and quantify the effect of noise on the system measurement. An adaptation of the Methodology defined by Hummel et al. [8] has been used.

A measurement plate was designed and manufactured to perform the evaluation tests. A $8 \times 9$ grid of holes was precisely drilled for position measurements. The tip of the instrument was placed in the centre of each position using a custom-made support and its $\mathrm{X}, \mathrm{Y}, \mathrm{Z}$ coordinates were computed (Figure 2a).

A second platform was developed to analyze the inclination accuracy of the system. It has 13 positions to place the instrument at intervals of 7.5 degrees, always keeping the tip fixed at the same position (Figure 2b).

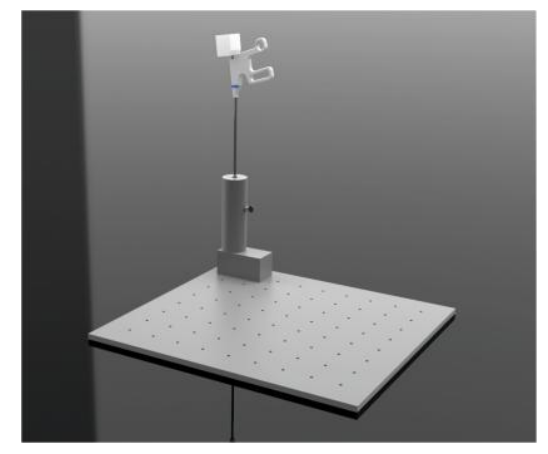

a

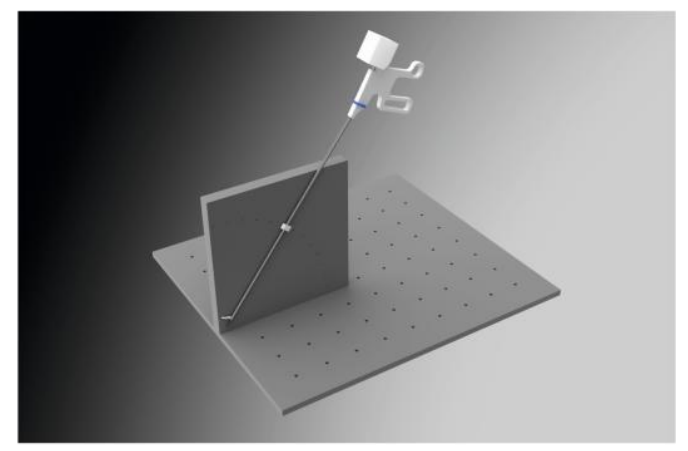

b

Fig. 2. Design of the measurements platforms to evaluate the positional accuracy (a) and the inclination accuracy (b).

The tip of the laparoscopic instrument was placed during 10 seconds at each position of the measurement platforms. The fluctuation error was evaluated by statistical analysis of position measurements. The root mean square error (RMSE) of positional coordinate was calculated at each laparoscopic instrument position.

Relative position errors were computed by comparing the Euclidean distances reported by the tracking system to the known physical distances on the measurement 
platforms. All the possible distances of 50, 100, 150, 200, 250, 300, and $350 \mathrm{~mm}$ were analyzed for the first measurement platform. In addition, the accumulated distance error was computed by means of obtaining the distances from the first position of the measurement plate $P(i, 1)_{i=1,2, \ldots, 8}$ to the other column positions $P(i, j)_{j=1,2, \ldots, 8}$, and comparing them with the real distances on the plate.

\section{Results}

The system demonstrates stable fluctuation error over the entire volume at $20 \mathrm{~Hz}$ (Figure 3). It decreases slightly with the distance and has its highest value (3.868 $\mathrm{mm})$ at the point $(5,7)$ of the measurement plate.

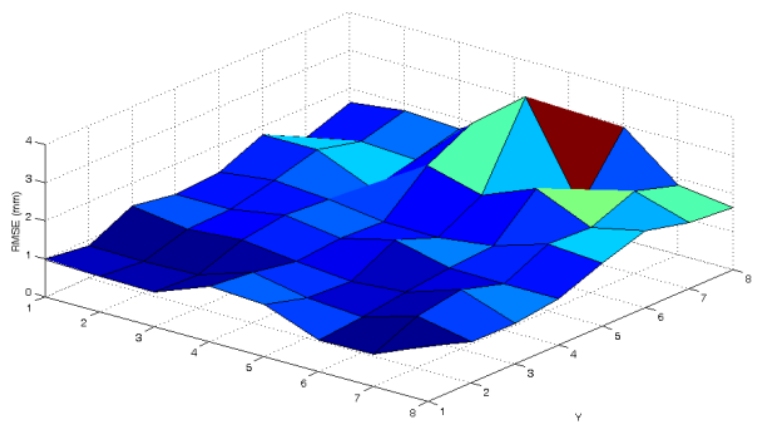

Fig. 3. System accuracy error (RMSE). X and Y label the position of the laparoscopic instrument on the platform, and $\mathrm{Z}$ indicates the resulting fluctuation error.

The results for the relative position errors were $1.776 \pm 1.675 \mathrm{~mm}, 1.817 \pm 1.762$ $\mathrm{mm}, 1.854 \pm 1.740 \mathrm{~mm}, 2.455 \pm 2.164 \mathrm{~mm}, 2.545 \pm 2.496 \mathrm{~mm}, 2.764 \pm 2.342 \mathrm{~mm}$, $2.512 \pm 2.493 \mathrm{~mm}$ for distances of $50,100,150,200,250,300$, and $350 \mathrm{~mm}$, respectively. The system is more accurate at shortest distances. However, it presents no significant differences with increasing distances.

In general terms, the accumulated distance error increases with the measured distance (Figure 4). The maximum accuracy of the tracking system is at $150 \mathrm{~mm}$ and the minimum at $350 \mathrm{~mm}$, with $7.800 \mathrm{~mm}$ of highest deviation. 


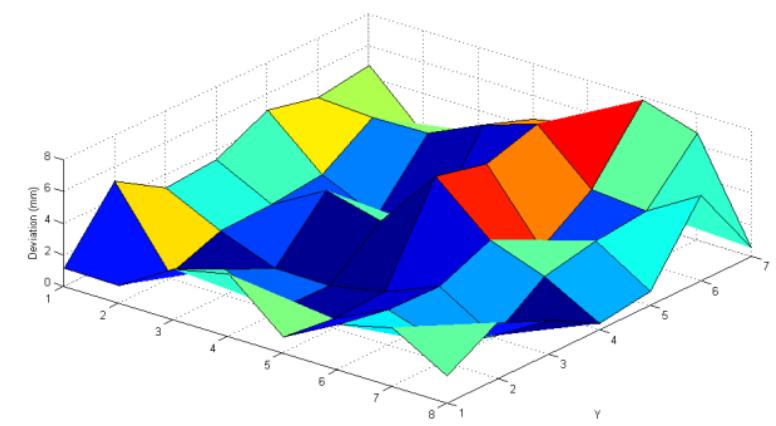

Fig. 4. Accumulated distance error. The error increases with regard to the distance from the first row of the measurement plate.

Table 1. Fluctuation error and accumulated distance error in computing the laparoscopic instrument tip position at different degrees of inclination.

\begin{tabular}{lll}
\hline Degree & RMSE $(\mathrm{mm})$ & $\begin{array}{l}\text { Accumulated } \\
\text { distance }(\mathrm{mm})\end{array}$ \\
\hline 90.0 & 0.568 & - \\
82.5 & 0.589 & 1.807 \\
75.0 & 0.688 & 1.136 \\
67.5 & 0.756 & 1.382 \\
60.0 & 0.640 & 1.328 \\
52.5 & 0.592 & 1.014 \\
45.0 & 0.692 & 0.651 \\
37.5 & 0.615 & 1.988 \\
30.0 & 0.431 & 1.907 \\
22.5 & 0.532 & 0.581 \\
15.0 & 0.737 & 1.294 \\
7.5 & 0.880 & 2.775 \\
\hline
\end{tabular}

The tracking system was able to track markers from 90 to 7.5 degrees. The maximum fluctuation error is at 7.5 degrees and also its highest accumulated distance error (Table 1).

\section{Discussion}

Third generation trackers offer some advantages over trackers of earlier generations. They are fully passive and use available visible illumination to identify targets. And, in general, they are more affordable. This kind of tracking systems can be compared favourably with magnetic tracking devices since they do not present metal interferences from objects (including the instrument) near the working area. 
The presented design adapts a third generation optical pose tracker (MicronTracker $\left.{ }^{\circledR}\right)$ to laparoscopy. It has not wires hanging from the instruments, allowing users natural movements and hardly any noticing weight differences. Nevertheless, this system has some challenges that need to be addressed.

As all optical trackers, this system needs a clear line of sight between markers and the camera system. This problem could be addressed using other support methods like inertial trackers or tracking methods based on the endoscopic image.

The instrument inclination covered by the system is wide (from 90 to 7.5 degrees). However, errors in the computed position of the instrument tip among different inclinations are high. This leads to different results for the same position of the tip when the inclination of the instrument is changed.

Occasionally, the system reports some peak values during the tracking process of a marker and consequently in the instrument tip position, decreasing the accuracy of the tracking results. It could be reduced applying filtering or thresholding techniques.

Errors are stable on the entire workspace and they do not present significant differences with increasing distances. As was reported by other researchers, this tracking system shows low accuracy [9] and its robustness is sensitive to illumination conditions as well as to the velocity and the orientation [10]. Some important factors to have in mind are the design and position of the markers. Placing the markers nearer the instrument tip could increase the accuracy in computing its position; however, this prevents the correct use of the instrument.

As future work, to increase the system's accuracy, the design of the markers will be enhanced. Also the human factor will be reduced as much as possible during the calibration processes in order to decrease errors during the computation process of the transformation matrices.

Comparing the results with other tracking systems for technical skills assessment and/or image-guided applications. Hummel et al. [8] compared the accuracy of two electromagnetic tracking systems, Aurora (Northem Digital Inc.; ON, Canada) and microBIRD (Ascension; VT, USA). Both systems reported higher accuracy $(0.96 \pm 0.68 \mathrm{~mm}$ and $1.14 \pm 0.78 \mathrm{~mm})$ for distances of $50 \mathrm{~mm}$, but it was lower for longer distances. Another work [11] obtained better results for relative position error using a Polhemus Isotrack (Inition; London, UK) in a similar experimental setting as the current study.

Regarding other commercial optical trackers, the Polaris optical tracking system (Northem Digital Inc; ON, Canada), which is currently used clinically, provides accuracy $<0.25 \mathrm{~mm}$ for a $240 \times 156 \times 131 \mathrm{~cm}$ of field of measurement. With respect to tracking systems based on endoscopic images, Zhang and colleagues [12] reported a mean position error of $0.935 \pm 0.779 \mathrm{~mm}, 9.341 \pm 2.048 \mathrm{~mm}$, and $1.783 \pm 0.744 \mathrm{~mm}$ for $\mathrm{X}, \mathrm{Y}$, and Z coordinates. A RMSE of $9.28 \mathrm{~mm}$ was obtained for Cano et al. [13]. However, these techniques have some difficulties with real time execution.

In this paper we have presented a system design to apply a third generation optical pose tracker to laparoscopic practice. The system can be used with real laparoscopic instruments and it allows users to grip and use the instruments in a natural way. Evaluation tests show stable but low positional accuracy to track the laparoscopic instrument tip. The reported results of the proposed system could be enough for objective assessment tasks based on instrument motion, however tracking systems are an 
essential component of any image-guided intervention and its accuracy is a critical issue. For this reason it is important to perform a comprehensive evaluation of the tracking system before its implementation in any clinical environment. In order to establish the clinical viability of this system, some reported limitations will be addressed and new studies of robustness will be performed.

\section{Acknowledgements}

This study was supported in part by the Plan Regional de Invstigación, Consejería de Empleo, Empresa e Innovación, Gobierno de Extremadura and the European Social Found (PRE08057).

\section{References}

1. Sánchez-Margallo J.A., Sánchez-Margallo F.M., Pagador J.B., Gómez E.J., SánchezGonzález P., Usón J., Moreno J.: Video-based assistance system for training in minimally invasive surgery. Minim Invasive Ther Allied Technol. 20, 197-205 (2011).

2. Sánchez-Margallo F.M., Díaz-Güemes I., Pérez F.J., Sánchez M.A., Loscertales B., Usón J.: Preliminary results with a training program for thoracoscopic atrial fibrillation therapy. Surg Endosc. 23, 1882-6 (2009).

3. Moyano-Cuevas J.L., Sánchez-Margallo F.M., Sánchez-Peralta L.F., Pagador J.B., Enciso S., Sánchez-González P., Gómez-Aguilera E.J., Usón-Gargallo J.: Validation of SINERGIA as training tool: a randomized study to test the transfer of acquired basic psychomotor skills to LapMentor. Int J Comput Assist Radiol Surg. 6, 839-46 (2011).

4. Halsted W.S.: The training of surgeons. Johns HopkingsHosp Bull. 15, 267 (1904).

5. van Hove P.D., Tuijthof G.J.M., Verdaasdonk E.G.G., Stassen L.P.S., Dankelman J.: Objective assessment of technical surgical skills. Br J Surg. 97, 972-87 (2010).

6. Oropesa I., Sánchez-González P., Lamata P., Chmarra M.K., Pagador J.B., SánchezMargallo J.A., Sánchez-Margallo F.M., Gómez E.J.: Methods and tools for objective assessment of psychomotor skills in laparoscopic surgery. J Surg Res. 171, e81-e95 (2011).

7. Perrin D.P., Vasilyev N.V., Novotny P., Stoll J., Howe R.D., Dupont P.E., et al.: Image guided surgical interventions. Curr Probl Surg. 46, 730-66 (2009).

8. Hummel J.B., Bax M.R., Figl M.L., Birkfellner W.W.: Design and application of an assessment protocol for electromagnetic tracking system. Med Phys. 32, 2371-9 (2005).

9. Clarke J.V., Deakin A.H., Nicol A.C., Picard F.: Measuring the positional accuracy of computer assisted surgical tracking systems. Comput Aided Surg. 15, 13-8 (2010).

10. Maier-Hein L., Franz A., Meinzer H-P., Wolf I.: Comparative assessment of optical tracking systems for soft tissue navigation with fiducial needles. Proceedings of SPIE. 6918 (2008).

11. Pagador J.B., Sánchez L.F., Sánchez-Margallo J.A., Bustos P., Moreno J., SánchezMargallo F.M.: Augmented reality haptic (ARH): an approach of electromagnetic tracking in minimally invasive surgery. Int J CARS. 6, 257-63 (2011).

12. Zhang X., Payandeh S.: Application of Visual Tracking for Robot-Assisted Laparoscopic Surgery. J Robot Syst. 19, 315-28 (2002).

13. Cano A.M., Gayá F., Lamata P., Sánchez-González P., Gómez E.J.: Laparoscopic Tool Tracking Method for Augmented Reality Surgical Applications. LNCS. 5104, 191-6 (2008). 